\title{
Morphological changes of neurons involved in long-term memory in day-old chicks hatching from hypomagnetic field space
}

\author{
Xuebin Wang ${ }^{1,2}$, Guang-Zhe Lin ${ }^{1}$, Junfeng $\mathrm{Li}^{2}$, Xu Zhang ${ }^{2}$, Muling $\mathrm{Xu}^{2}$, Dongfeng $\mathrm{Li}^{3}$, \\ Jinchang Jiang ${ }^{1}$, Yan-Lin Sun ${ }^{4}$, Soon-Kwan Hong ${ }^{5}$, Hyun-Yong Jang ${ }^{6}$ and Jong-Suh Shin ${ }^{6 *}$ \\ ${ }^{1}$ School of Life Science, Linyi University, Linyi 276005, China. \\ ${ }^{2}$ Laboratory of Visual Information Processing, Institute of Biophysics, Chinese Academy of Science, Beijing 100101, \\ China. \\ ${ }^{3}$ School of Life Science, South China Normal University, Guangzhou 510631, China. \\ ${ }^{4}$ School of Life Sciences, Ludong University, Yantai, Shandong 264025, China. \\ ${ }^{5}$ Department of Bio-Health Technology, College of Biomedical Science, Kangwon National University, 200-701, Korea. \\ ${ }^{6}$ Department of Animal Resource Science, College of Animal Life Sciences, Kangwon National University, 200-701, \\ Korea.
}

Accepted 10 August, 2012

\begin{abstract}
Hatched from natural geomagnetic conditions, 1-day-old chicks were stimulated with Methyl Anthranilate (MeA) and labeled as the control group (CG). We found that the line density of dendritic spines (LDds) from the neurons in the memory-related nuclei [Intermediate Medial Hyperstriatum Ventrale (IMHV) and lobus parolfactorius (LPO)] was increased by $38.7 \%$, and the total dendritic lengths (TLds) of individual neurons in IMHV were increased by 37.1 to $45.4 \%$. In addition, the average LDds of individual neurons in imprinted chicks (ICs) were increased by $10.8 \%$, and the neurons that had long dendrites were increased by $50 \%$. In contrast, in the experimental group (EG), where chicks were hatched from hypomagnetic field, the average LDds of each neuron in IMHV and LPO of the native chick (NC) were similar to the NC of CG. After a $12 \mathrm{~h}$ exposure to MeA, the LDds from IMHV and LPO of the no-imprinted chick (n-IC) and IC were similar to NC but decreased by $17.4 \%$ to the $n-I C$ and IC of CG. Furthermore, the average TLd of single neurons from the memory nuclei in NC, $n-I C$ and IC were significantly decreased by $30.9 \%$. However, the LPOs were not different in n-IC. These results indicate that if the natural geomagnetic environment is disrupted, the development of the dendritic spines from the neurons in the related memory nuclei is unchanged, but the hyperplasia of the dendritic spines in the neurons involved in long-term memory was ablated by MeA. Further, the development and growth of dendrites were significantly reduced.
\end{abstract}

Key words: Hypomagnetic field space, day-old chicks, memory-related nuclei (Medial Hyperstriatum Ventrale and lobus parolfactorius), neuron morphology.

\section{INTRODUCTION}

The biological effects of a hypomagnetic field space (HMFS), where the magnetic intensity was reduced below $1000 \mathrm{nT}(1 \mu \mathrm{T})$, had been studied extensively by the former Soviet Russia in the 1960 to 1970s. Their

${ }^{*}$ Corresponding author. E-mail: jsshin@kangwon.ac.kr. studies focused on the effects of the HMFS on the development and growth of various organisms. However, the studies that examined the influence of HMFS on the function of the central nervous system were limited to the measurement of the critical flicker fusion rate (Dubrov, 1978). To date, studies have examined the effects of HMFS on the learning of the birdsong and on the levels of neurotransmitters in the rat brain (Jiang et al., 1998; Li 
et al., 2001), and focused on the effects of weak magnetism, where the magnetic intensity was similar to the geomagnetism on learning and memory (Lovely et al., 1992); studies on the biological effects of extremely low intensity magnetism (below $1 \mu \mathrm{T}$ ) have not been conducted. Using a previous model for creating the HMFS (Jiang et al., 2003), we showed that long-term memory was impaired in a one-trial passive avoidance task in 1-day-old chicks that hatched from the HMFS (Wang et al., 2003), and we also found that learning and memory were significantly impaired in Drosophila that were continuously housed in the HMFS (Zhang et al., 2004).

These results indicate that if natural geomagnetism is abolished during the development of the embryo, the structure and function of the neurons involved in learning and memory will be altered.

Since Gibbs and $\mathrm{Ng}$ (1976) suggested a three-term memory model for the one-trial avoidance task of 1-dayold chicks many studies in the neuroscience and cognitive science fields have focused extensively on memory and increased our understanding of the neural mechanisms involved in the formation of short-, mediumand long-term memory.

The areas of the brain involved in the formation of memories in chicks are the intermediate medial hyperstriatum ventrale (IMHV) and the lobus parolfactorius (LPO) (Rose, 1991; Squire et al., 1993). In addition, the formation of long-term memory is important in the synthesis of $\alpha$-tubulin and glycoprotein (Scholey et al., 1992, 1993 Rose, 1995), and it is closely related to morphological changes in neurons (such as the density of dendritic spines, quantity of vesicles, and the density and height of synapses (Stewart et al., 1992; Stewart and Rusakov, 1995; Rusakov et al., 1993; Rose and Stewart, 1999; Ünal et al., 2002). Long-term memory initially forms in the IMHV and is then stored in the LPO (Rose, 1991; Tiunova et al., 1998). Recent studies have shown that the N-Methyl-D-aspartate (NMDA) receptor (NMDAR) and the a-Amino-3-hydroxy-5-methyl-4-isoxazolepropionic acid (AMPA) receptor (AMPAR) in the IMHV and LPO in chicks are important in the origination of memory formation and retention (Soderling and Derkach, 2000). In addition, the regulation of glutamate and the activation of NMDAR can increase $\mathrm{Ca}^{2+}$ concentration within cells, which affects synaptic structures (such as prolonging, shortening and collapsing the dendritic spines (Segal et al., 2000). Therefore, we examined the morphological changes in neurons from 1-day-old chicks that were hatched from a hypomagnetic field and had long-term memory impairments. This comparative study on 1-dayold chicks hatched in a natural geomagnetic field and a hypomagnetic field showed that during the development of the chick embryo, the hypomagnetic field does not affect the growth of the dendritic spines. However, in the onetrial avoidance task, hyperplasia of the dendritic spines was reduced, and the development and growth of the dendrites were significantly inhibited.

\section{MATERIALS AND METHODS}

The compensated hypomagnetic field space comes from 3 pairs of Helmholtz coils intersecting vertically one another whose diameters are $2.01,1.80$ and $1.61 \mathrm{~m}$ and compensating the values of magnetic field in vertical, north and south, and east and west, respectively. The value of residual magnetic field is in 100 to 680 $\mathrm{nT}$. In the hypomagnetic field space, a HJ-LC112 intelligent nonferromagnetic incubator is arranged (Jiang et al., 2003).

The 1-day-old chicks that were hatched from the natural geomagnetic field in the control group (CG) and the hypomagnetic field in the experimental group (EG) were tested in a one-trial avoidance task using MeA (Methyl Anthranilate (Wang et al., 2003). For all groups, three naive chicks (NC), three no-imprinted chicks (n-IC) and three imprinted chicks (IC) were chosen, and the neuronal morphology was observed $12 \mathrm{~h}$ after being trained.

The Golgi silver stain has been widely used to study neuronal morphology (Jiang et al., 2003; Peters and Kaiserman-Abramof, 1970; Rusakov and Stewart, 1995; Sojka et al., 1995). According to the improved method, we were able to localize each neuron to the memory-related nuclei. The anesthetized chicks were perfused and fixed with physiological saline and 10\% formalin for $20 \mathrm{~min}$, and then the brains were removed. Following Youngren's brain atlas (Youngren and Phillips, 1978), the tissue masses $(1 \times 1.5 \times 2 \mathrm{~mm})$ of the right and left IMHVs and LPOs were excised, and they were then post-fixed for 1 week at room temperature. Next, the tissues were washed with DW and then immersed in $0.75 \%$ silver nitrate for 6 days. After being washed with $40 \%$ alcohol for $40 \mathrm{~min}$, the tissues were dehydrated sequentially using 70, 80 and $95 \%$ alcohol.

The tissues were immersed in alcohol for approximately $2 \mathrm{~h}$, dipped into a mixture of ether and alcohol $(1: 1)$ for $3 \mathrm{~h}$, and infused with 4 and $8 \%$ collodion for 24 and $1 \mathrm{~h}$, respectively. The tissues were indurated by $70 \%$ alcohol and sliced at $20 \mu \mathrm{m}$. The slices were dehydrated sequentially using 70, 80 and $95 \%$ alcohol. After being cleared by creosote for $5 \mathrm{~min}$, the slices were mixed sufficiency with oil of turpentine to dissolve the creosote. Next, the slices were placed onto slides, covered with Canada gum, and airdried for 3 weeks.

The dried slices were detected using an inverted microscope (Olympus), and photos were taken using a micro-CCD photography system (Cold Spring Harbor Corp.). The photo managing and length measuring were analyzed using Image-pro Plus software.

Thirty neurons were selected from each nucleus, and a clear, medial segment of dendrites was chosen from the neurons. The sampling length of the dendrite (SLd) and the number of dendritic spines (Nds) were measured using a high-fold microscope lens. The line density of the dendritic spines (LDds) was calculated using the formula: LDds $=\mathrm{Nds} / \mathrm{SLd}$. Furthermore, for the nuclei related to memory, the total length of the dendrites (TLds) of the neurons that were easily identifiable was measured.

\section{RESULTS}

\section{LDds and TLd in the control group}

Figure 1 shows the neuronal morphology in the memory related nuclei of the $\mathrm{NC}, \mathrm{n}-\mathrm{IC}$ and $\mathrm{IC}$ from the control group. In Figure 1A-1-4, 1B-1-4 and 1C-1-4, the atramentous body and the dendritic spines of the stellate neurons can be clearly observed, which was consistent with Stewart et al. (1992). In addition, the branches from 


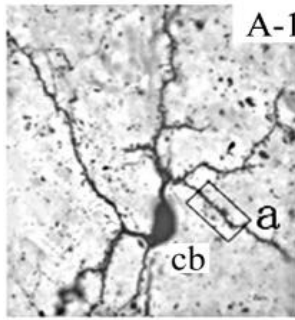

B-1
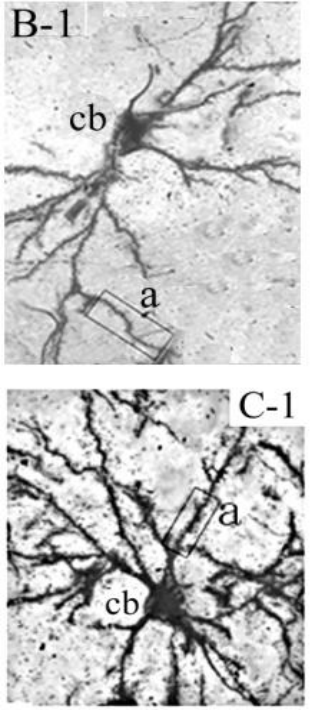

$\mathrm{C}-1$
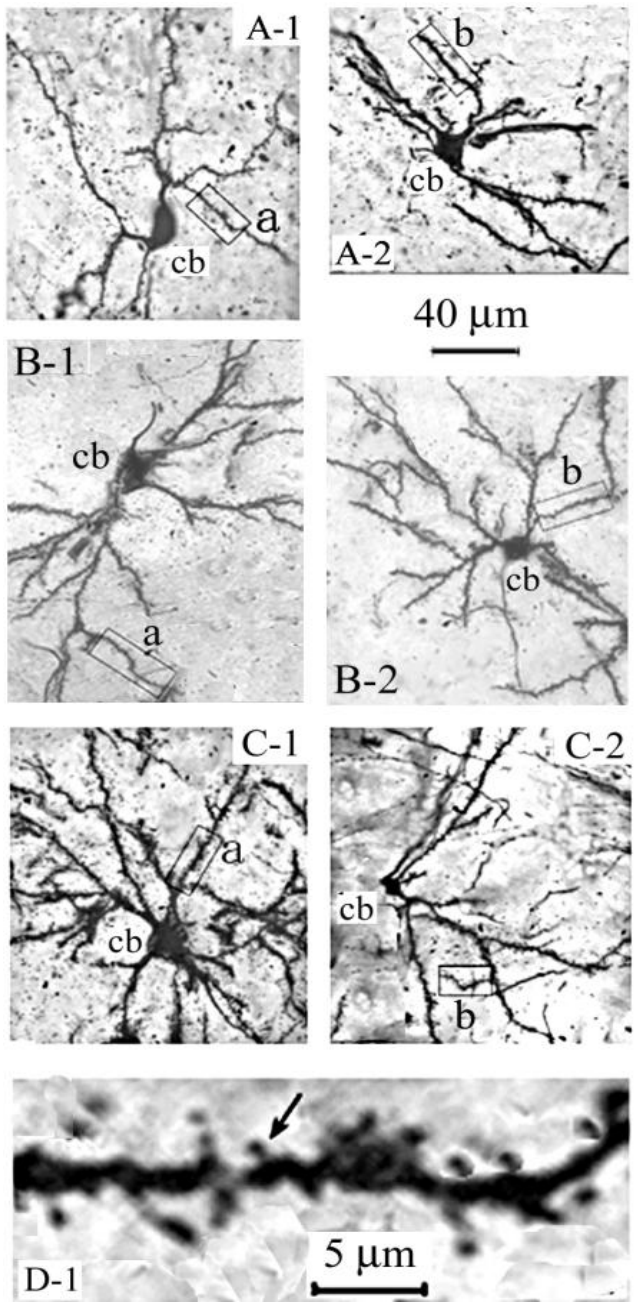

$40 \mu \mathrm{m}$

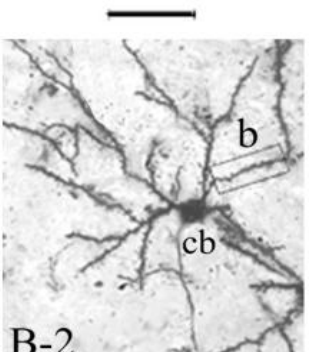

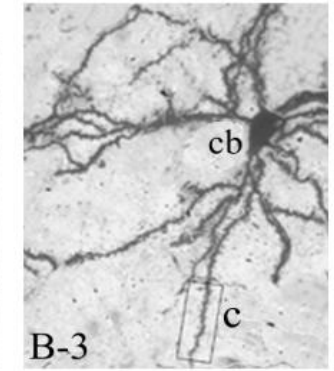
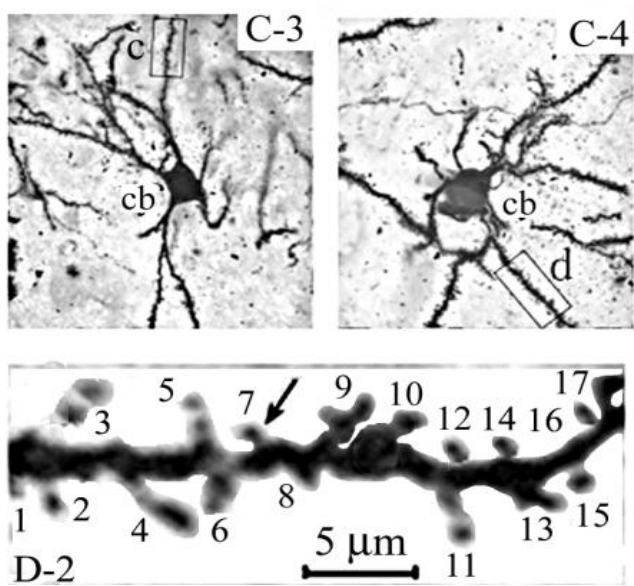

Figure 1. The morphology of neurons and dendritic spines in the memory-related nuclei of 1day-old chicks from the control group. The neurons of the R-IMHV, R-LPO, L-IMHV and L-LPO in the NC (A-1-4), n-IC (B-1-4) and IC (C-1-4), respectively; cb: cell body; a-d: sampling segment of the dendrite. D-1 is a magnification of from A-1. D-2 shows D-1, in which the surrounding background of the dendritic spines was removed. The arrow shows a dendritic spine.

the central neurons in the memory-related nuclei in the NC were significantly less than in the $\mathrm{n}-\mathrm{IC}$ and IC, and the R-IMHV of the IC was the most dense among them (Figure 1C-1). This was consistent with the TLd data (Figure 3).

Using a high-fold lens, the dendritic spines of the sample segments (a-d) in Figure 1A-1-4, 1 B-1-4 and C1-4 can be seen by adjusting the fine focus on the microscope. Because of the 3-dimensional structure of the dendritic spines (Rusakov and Stewart, 1995; Sojka et al., 1995), the most representative image depicting the majority of the dendritic spines was chosen.

Of the 360 SLds examined from the control group, the shortest was $24.9 \mu \mathrm{m}$, and the longest was $119.8 \mu \mathrm{m}$; the majority was found to be from 30 to $80 \mu \mathrm{m}$, which accounted for $85.8 \%$ (309/360).

In Figure 1A-1-4, the lengths of the SLd were 29, 37, 37 and $33 \mu \mathrm{m}$, respectively, with the number of corresponding dendritic spines (Nds) in the segments being 17, 25, 22 and 21 (Figure 1D-2), and the LDds being 0.59, $0.68,0.60$ and $0.64 \mu \mathrm{m}$, respectively. Similarly, in Figure 1B-1-4, the SLds in a-d were 42, 40, 44 and $51 \mu \mathrm{m}$, respectively; the Nds were $35,32,37$ and 40 , and the LDds were $0.83,0.80,0.84$ and $0.78 \mu \mathrm{m}$, respectively. In Figure 1C-1-4, the SLds in a-d were $33,29,30$ and 34 $\mu \mathrm{m}$, respectively; the Nds were $30,26,28$ and 28 , and the LDds were $0.91,0.90,0.93$ and $0.82 \mu \mathrm{m}$, respectively. The LDds of the neurons were increased in the central memory-related nuclei in the 1-day-old chicks that were exposed to MeA ( $\mathrm{n}-\mathrm{IC}$ and IC) compared to the NC.

The distribution of the perpetual percentage of LDds and TLd (PFds and PFd) of the neurons from the left and the right of the IMHV and LPO of the NC, n-IC and IC from the control group is displayed in Figure 2(1) and (2). 

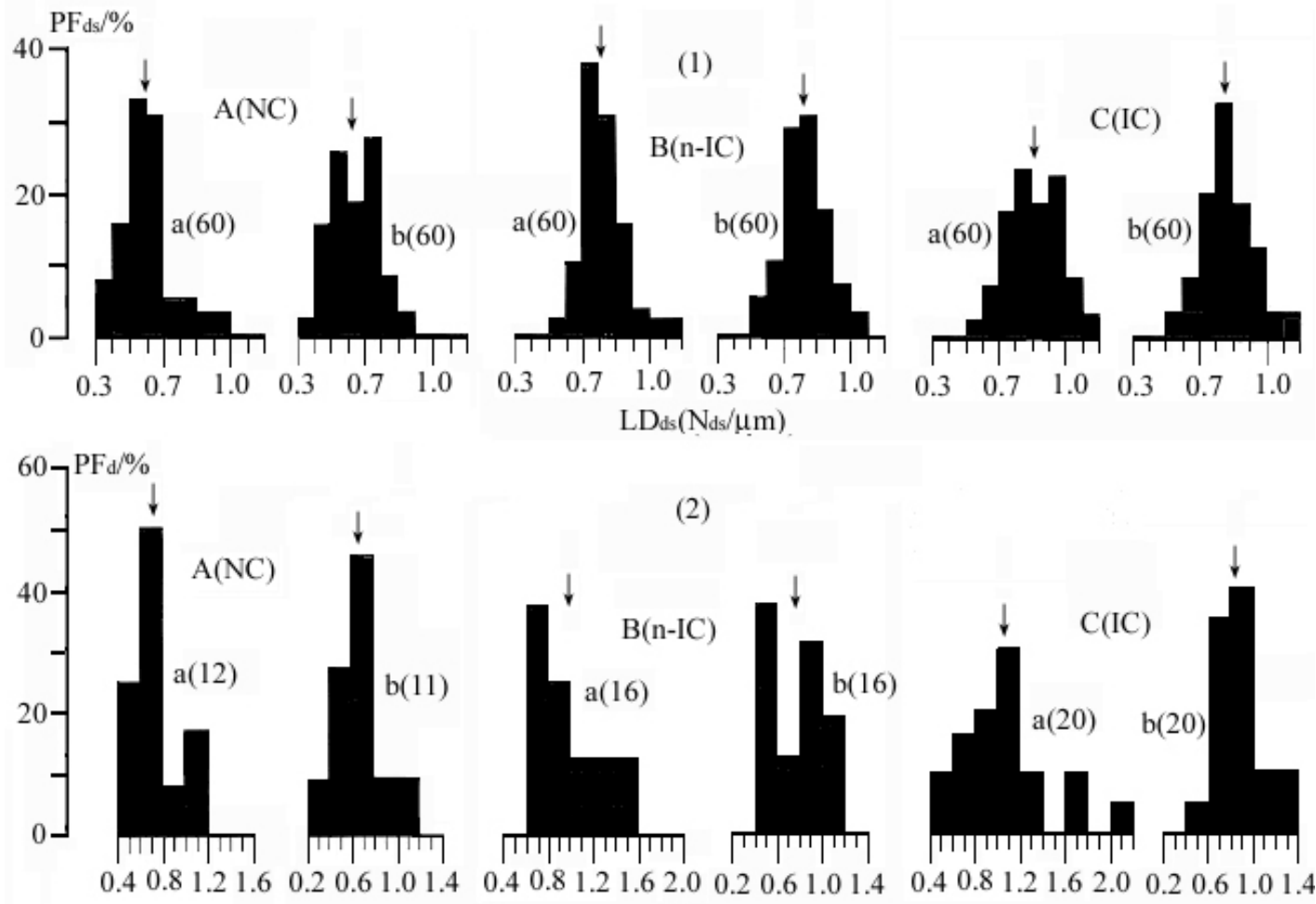

$\mathrm{TLd} / \mathrm{mm}$

Figure 2. The distribution of LDds and TLd frequency of the percentage of the neurons in the memoryrelated nuclei of 1-day-old chicks from the control group (1 and 2). a, IMHV; b, LPO. The arrowheads show the mean value, and the numerals in brackets show the number of samples.

In Figure 2(1)-A-a and $b$, as the control group, the main distribution of the neuronal LDds frequency in the left and right side of the IMHV and LPO in the NC were 0.4 to 0.7 $\mu \mathrm{m}$ (accounting for $76.7 \%$ ) and 0.4 to $0.8 \mu \mathrm{m}$ (accounting for $85.0 \%)$, and the mean values were $0.60 \pm 0.15 \mu \mathrm{m}$ (60) and $0.65 \pm 0.16 / \mu \mathrm{m}(60)$, respectively. There was no significant difference between the two nuclei.

Similarly, the main distribution of the neuronal LDds frequency in the left and right side of the IMHV and LPO in the $\mathrm{n}-\mathrm{IC}$ were both about 0.6 to $1.0 \mu \mathrm{m}$, which accounted for 91.7 and $85.0 \%$, respectively, and the mean values were $0.83 \pm 0.12 \mu \mathrm{m}(60)$ and $0.83 \pm 0.14$ $\mu \mathrm{m}(60)$, respectively. There was no significant difference between nuclei. However, compared to the NC, the mean values increased significantly by $37.9 \%(t=9.025>t$ $0.01, \mathrm{df}=118)$ and $28.6 \%(\mathrm{t}=6.945>\mathrm{t} 0.01$, $\mathrm{df}=118)$, respectively.

In addition, the main distribution of the neuronal LDds frequency in the left and right side of the IMHV and the LPO in the IC was 0.7 to $1.1 \mu \mathrm{m}$, which accounted for 80.0 and $81.7 \%$, respectively, the mean values were 0.92 $\pm 0.15 \mu \mathrm{m}(60)$ and $0.87 \pm 0.15 \mu \mathrm{m}(60)$, respectively, and there was no significant difference. However, compared to the NC, the mean values were increased significantly by $52.8 \%(\mathrm{t}=10.432>\mathrm{t} 0.01, \mathrm{df}=118)$ and $33.8 \%(\mathrm{t}=$ $7.907>$ t $0.01, \mathrm{df}=118)$, respectively. Further, the LDds from the two sides of the IMHV in the IC increased, on average, by $10.8 \%(\mathrm{t}=3.621>\mathrm{t} 0.01$, $\mathrm{df}=118)$ compared to the $\mathrm{n}-\mathrm{IC}$.

Therefore, the neuronal LDds frequency of the central memory-related nuclei in the 1-day-old chicks treated with MeA ( $\mathrm{n}-\mathrm{IC}$ and IC) increased, on average, by $38.3 \pm$ $10.4 \%$ (4) compared to the NC.

As shown in Figure 2(2)-A-a and b, we found that in the control group, the main distribution of the TLd of the neurons from the left and right side of the IMHV and the LPO in the NC was 400 to $800 \mu \mathrm{m}$, which accounted for 75 and $72.5 \%$, respectively, and the mean values were $733 \pm 170 \mu \mathrm{m}$ (12) and $673 \pm 243 \mu \mathrm{m}$ (11), respectively. There was no significant difference between the two nuclei. The neurons with TLd over $1000 \mu \mathrm{m}$ accounted for 16.7 and $9.1 \%$, respectively.

Similarly, we showed in Figure 2(2)-B-a and b that the main distribution of the TLd of the neurons from the left and right side of the IMHV and the LPO in the $\mathrm{n}-\mathrm{IC}$ was 600 to $1000 \mu \mathrm{m}$ and 400 to $1000 \mu \mathrm{m}$, which accounted for 62.5 and $81.3 \%$, respectively, and the mean values were $1005 \pm 317 \mu \mathrm{m}(16)$ and $778 \pm 203 \mu \mathrm{m}$ (16). The TLd in the IMHV increased, on average, by $29.2 \%(\mathrm{t}=$ $2.409>\mathrm{t} 0.05$, df $=30$ ) compared to that in the LPO. The neurons with TLd over $1000 \mu \mathrm{m}$ accounted for 37.5 and $18.8 \%$, respectively. Moreover, the average TLd in the 

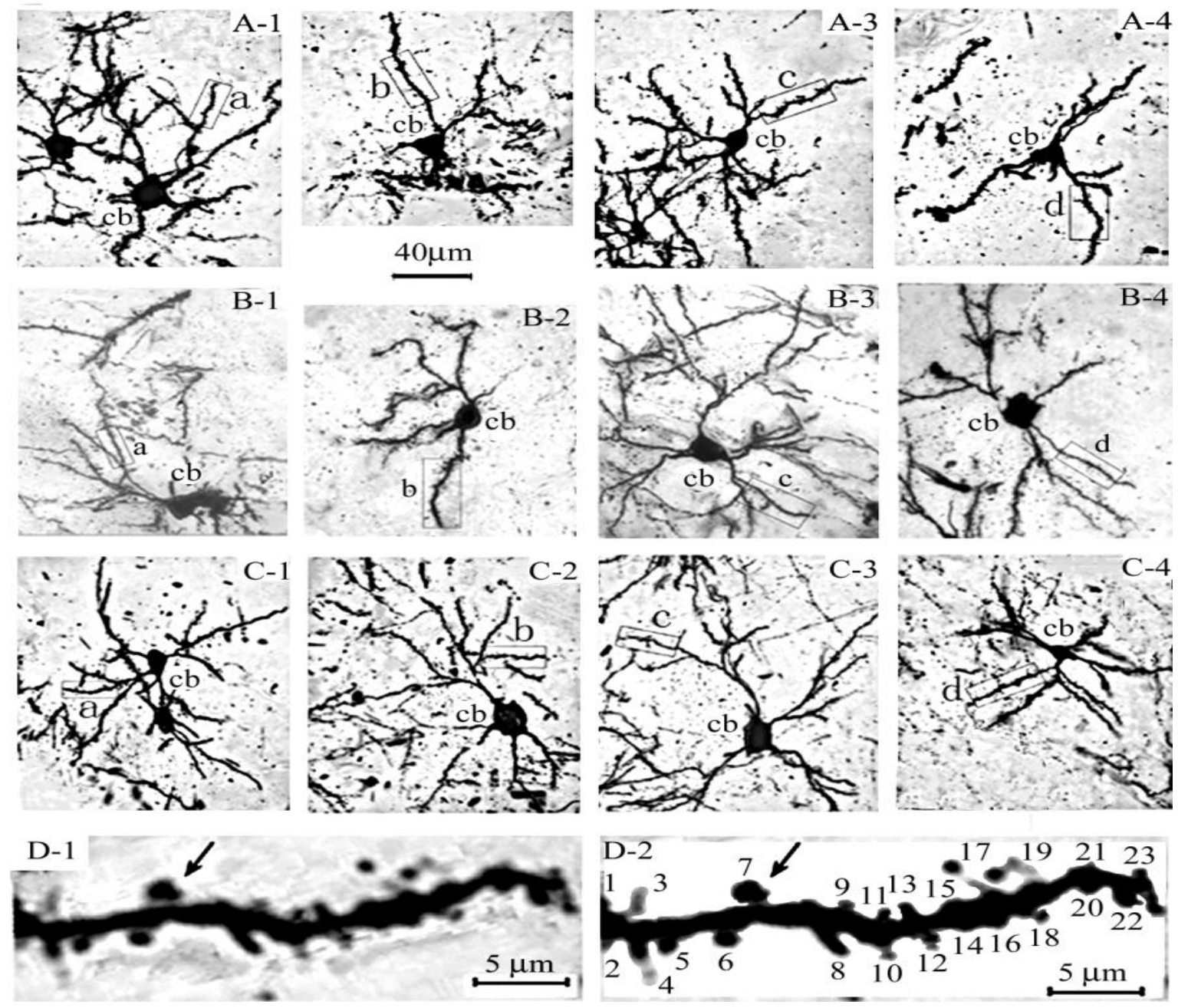

Figure 3. The morphology of the neurons and the dendritic spines in the memory-related nuclei of 1-day-old chicks in the experimental group. The neurons of the R-IMHV, R-LPO, L-IMHV and L-LPO in the NC (A-1-4), n-IC (B-1-4) and IC (C-1-4), respectively; cb, cell body; a-d, sampling segment of dendrite. D-1 shows the magnification of Figure 3A-1-a.; D-2 shows D-1 with the surrounding background of the dendritic spines removed. The arrow indicates a dendritic spine.

IMHV in the $\mathrm{n}-\mathrm{IC}$ increased significantly by $37.1 \%(\mathrm{t}=$ $2.687>$ t 0.02 , $d f=26$ ) compared to the NC.

As shown in Figure 2(2)-C-a and b, in the control group, the main distribution of the TLd of the neurons in the left and right side of the IMHV and the LPO in the IC was 600 to $1200 \mu \mathrm{m}$ and 600 to $1000 \mu \mathrm{m}$, which accounted for 65.0 and $80.0 \%$, respectively, and the mean values were $1066 \pm 386 \mu \mathrm{m}(20)$ and $851 \pm 196 \mu \mathrm{m}$ (20), respectively. The average TLd in the IMHV increased by $25.3 \%(\mathrm{t}=2.222>\mathrm{t} 0.05, \mathrm{df}=38)$ as compared to the LPO. The neurons with TLd over $1000 \mu \mathrm{m}$ in the IMHV and LPO accounted for 55.0 and $20 \%$, respectively. Moreover, the average TLd in the IMHV in the IC increased significantly by $45.4 \%(t=2.721>t 0.02$, df $=$ 30) compared to the NC. In contrast, there was no significant difference between the TLd in the IMHV in the $I C$ and the $\mathrm{n}-\mathrm{IC}$, but the neurons with TLd over $1000 \mu \mathrm{m}$ in the IC were 1.5 times more than in the $\mathrm{n}-\mathrm{IC}$.

\section{LDds and TLd in the experimental group}

Figure 3 shows the neuronal morphology in the memoryrelated nuclei isolated from the $\mathrm{NC}, \mathrm{n}-\mathrm{IC}$ and IC in the experimental group.

In Figure 3A-1-4, 3B-1-4 and 3C-1-4, the atramentous body and the numerous dendritic spines of the satellite neurons are clearly visible, which was similar to the control group? In contrast to the control group, the NC showed reduced neuronal dendritic branches in the memory-related nuclei compared to the $\mathrm{n}-\mathrm{IC}$ and IC.

Using the high-magnification lens, the dendritic spines sample segments (a-d) in Figure 3A-1-4, 3B-1-4 and 3C1-4 can be observed by adjusting the fine focus of the 

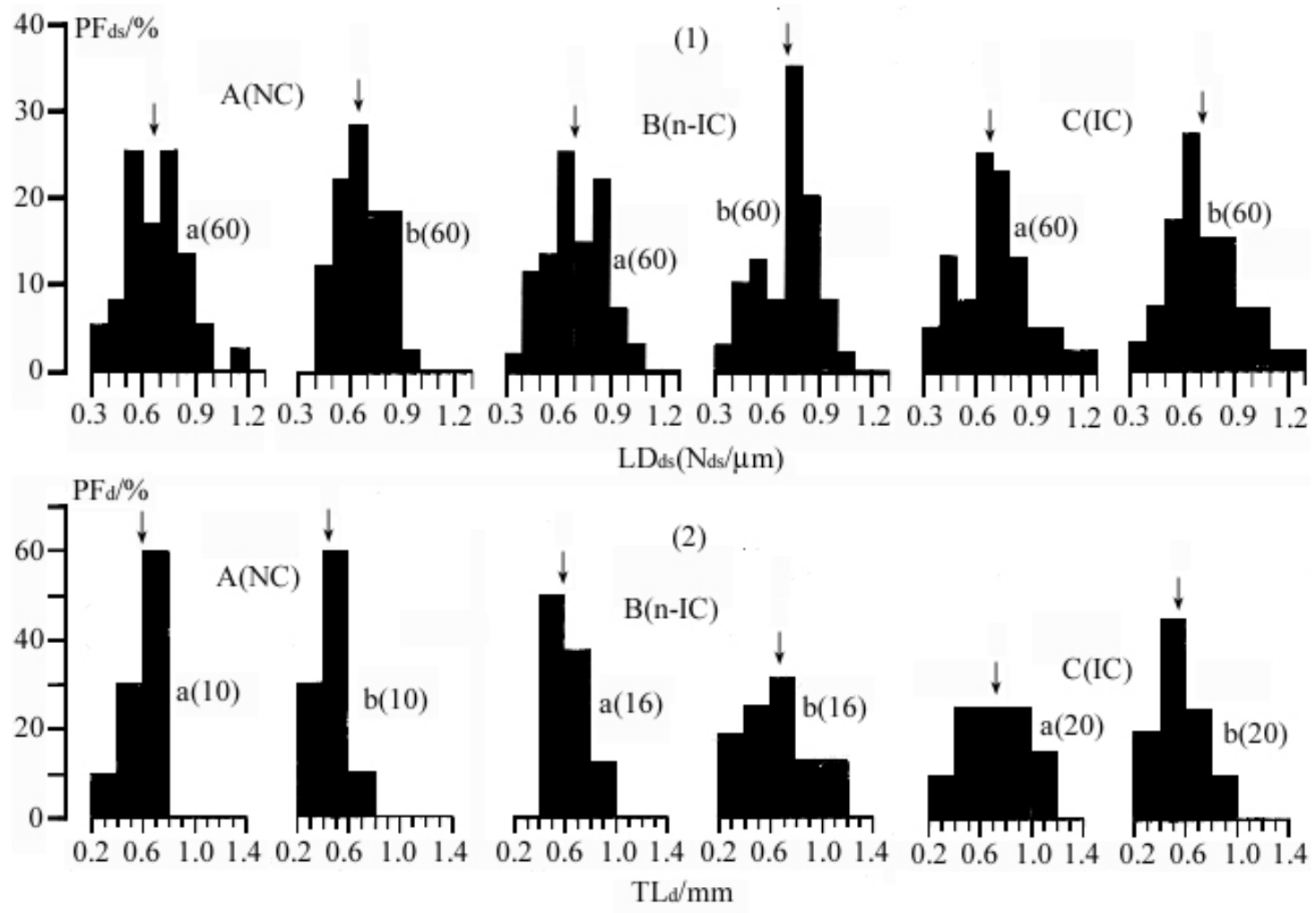

Figure 4. Distribution of the LDds and TLd frequency percentage of the neurons in the memory-related nuclei of 1-day-old chicks in the experimental group (1 and 2). a, IMHV; b, LPO. The arrowheads show the mean value, and the numerals in brackets show the number of samples.

microscope. Similar to the control group, the images are representative of the majority of visible dendritic spines. Moreover, some spines were in the developing stage (14 to 16,21 and 22). Of the 360 SLds examined from the experimental group, the shortest was $17.7 \mu \mathrm{m}$, and the longest was $120.5 \mu \mathrm{m}$; the majority was found to be between 30 to $70 \mu \mathrm{m}$, which accounted for $81.4 \%$ (293/360). In Figure 3A-1-4, the lengths of the SLd were $32,38,42$ and $35 \mu \mathrm{m}$, respectively, and the $\mathrm{Nds}$ in the segments were 23, 24, 28 and 22 (Figure 3D-2). Therefore, the LDds were $0.72,0.63,0.67$ and $0.63 \mu \mathrm{m}$, respectively. Similarly, in Figure 3B-1-4, the SLd in a-d were $25,52,39$ and $33 \mu \mathrm{m}$, respectively, the Nds were 18 , 35, 26 and 23, and the LDds were 0.72, 0.67, 0.67 and $0.70 \mu \mathrm{m}$, respectively. In Figure 3C-1-4, the SLds in a-d were $39,33,30$ and $34 \mu \mathrm{m}$, respectively; the Nds were 26, 24, 20 and 24, and the LDds were 0.67, 0.73, 0.67 and $0.71 \mu \mathrm{m}$, respectively. Compared to the NC, the LDds was not significantly different in the central memory-related nuclei from the 1-day-old chicks that had been treated with $\mathrm{MeA}$ ( $\mathrm{n}-\mathrm{IC}$ and IC).

The PFds and PFd of the neurons from the left and right side of the IMHV and the LPO of the NC, n-IC and IC in the experimental group are displayed in Figure 4(1) and (2).

In Figure 4, the main distribution of the neuronal LDds in the left and right side of the IMHV and the LPO in the NC of the experimental group was 0.5 to $0.9 \mu \mathrm{m}$ (accounting for $80.0 \%$ ) and 0.4 to $0.9 \mu \mathrm{m}$ (accounting for $98.3 \%$ ), and the mean values were $0.67 \pm 0.16 \mu \mathrm{m}(60)$ and $0.66 \pm 0.12 \mu \mathrm{m}(60)$, respectively. There was no significant difference between the two nuclei, which was similar to the NC in the control group.

Similarly, the main distribution of the neuronal LDds in the left and right sides of the IMHV and the LPO in n-IC was $0.40 .9 \mu \mathrm{m}$, which accounted for $86.7 \%$, and the mean values were $0.70 \pm 0.17 \mu \mathrm{m}(60)$ and $0.72 \pm 0.16$ $\mu \mathrm{m}(60)$, respectively. In addition, there was no significant difference between nuclei, and there was also no significant increase compared to the NC.

In addition, the main distribution of the neuronal LDds in the left and right side of the IMHV and the LPO in the IC were 0.4 to $0.9 \mu \mathrm{m}$ (accounting for $83.3 \%$ ) and 0.5 to 0.9 $\mu \mathrm{m}$ (accounting for $73.3 \%$ ), and the mean values were $0.70 \pm 0.20 \mu \mathrm{m}(60)$ and $0.72 \pm 0.20 \mu \mathrm{m}(60)$, respectively. Further, there was no significant difference between nuclei. There were no significant different in the mean values between the NC and the $\mathrm{n}-\mathrm{IC}$.

In Figure 4(2)-A-a and $b$, we found that in the experimental group, the main distribution of the TLd of the neurons in the left and right side of the IMHV and the LPO in the NC ranged from 400 to $800 \mu \mathrm{m}$ (accounting 

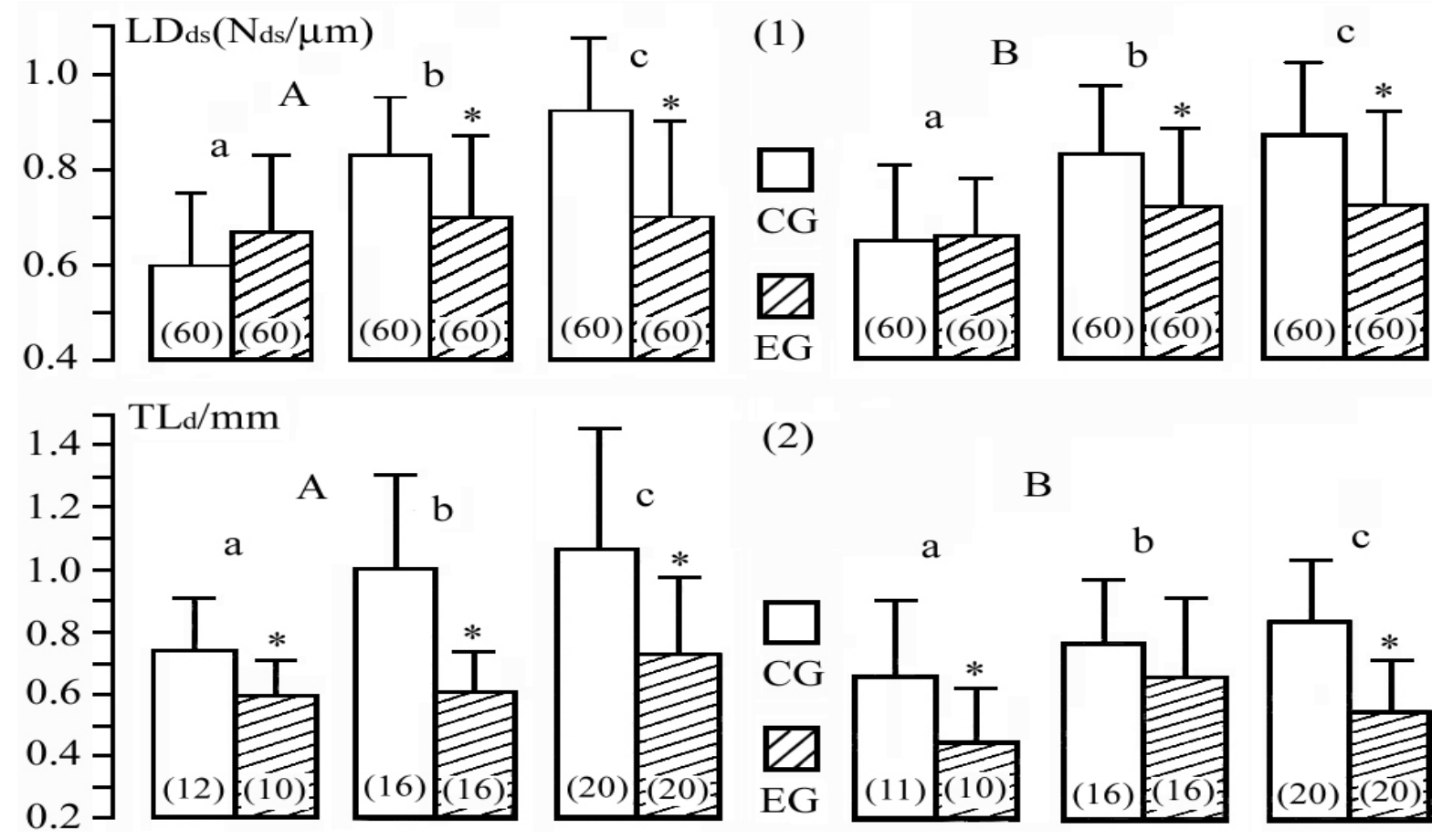

B

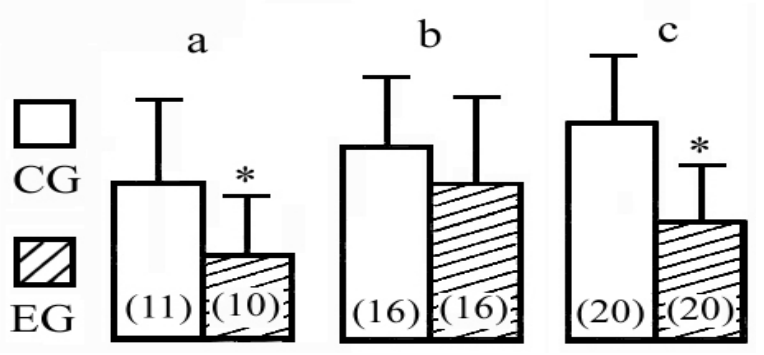

Figure 5. Comparison of the morphological features of the neurons in the memory-related nuclei between the experimental and control groups. (1), LDds, (2), TLd; A and B indicate the left and right side of the IMHV and LPO. a, $\mathrm{b}$ and $\mathrm{c}, \mathrm{NC}, \mathrm{n}-\mathrm{IC}$, and IC. The numerals in brackets represent the number of samples. ${ }^{*}$ Not significantly different $(P$ $<0.05)$.

for $90 \%$ ) and 200 to $600 \mu \mathrm{m}$ (accounting for $90 \%$ ), respectively, and the mean values were $603 \pm 110 \mu \mathrm{m}$ (10) and $462 \pm 168 \mu \mathrm{m}(10)$, respectively. The average TLd in the IMHV was increased significantly by $30.5 \%(\mathrm{t}=$ $2.222>$ t 0.05 , df $=18$ ) compared to the LPO, but the neurons with TLd over $1000 \mu \mathrm{m}$ were not found.

Similarly, as shown in Figure 4(2)-B-a and b, we demonstrated that the main distribution of the TLd of the neurons in the left and right side of the IMHV and the LPO in the n-IC of the experimental group was 400 to 800 $\mu \mathrm{m}$ (accounting for $85.7 \%$ ) and 200 to $600 \mu \mathrm{m}$ (accounting for $75 \%$ ), respectively, and the mean values were $607 \pm 132 \mu \mathrm{m}(16)$ and $670 \pm 253 \mu \mathrm{m}$ (16). Further, there were no significant differences between the TLd in the IMHV and the LPO. Moreover, the TLd in the IMHV in the $\mathrm{n}-\mathrm{IC}$ was not significantly different than the NC, whereas the average TLd in the LPO was significantly increased by $45.0 \%(t=2.295>t 0.05$, df $=24)$ as compared to the NC, and the neurons with TLd over 1000 $\mu \mathrm{m}$ accounted for $12.5 \%$.

In Figure 4(2)-C-a and b, the main distribution of the TLd of the neurons in the left and right side of the IMHV and the LPO in the IC was 400 to $1000 \mu \mathrm{m}$ (accounting for $75 \%$ ) and 200 to $800 \mu \mathrm{m}$ (accounting for 90\%), respectively, and the mean values were $734 \pm 253 \mu \mathrm{m}$ (20) and $560 \pm 175 \mu \mathrm{m}$ (20). In the IMHV in the NC, the neurons with TLd over $800 \mu \mathrm{m}$ were not found, but $40 \%$ were found in the IC. The mean value of the TLd in the IC increased by $21.7 \%$ compared to the NC. The number of neurons with TLd over $800 \mu \mathrm{m}$ were two times more than in the $\mathrm{n}-\mathrm{IC}$ and were significantly increased by approximately $20.9 \%(t=1.816>t 0.07, d f=34)$.

\section{Comparison between experimental and control groups}

The comparison between the LDds and TLd of the neurons found in the memory-related nuclei in the NC, nIC and IC in the experimental and control groups are demonstrated in Figure 5.

In Figure 5(1)-A and B, the LDds of the neurons in the IMHV and the LPO in the NC were not significantly different between groups; but both the $\mathrm{n}-\mathrm{IC}$ and IC were decreased significantly by $17.4 \pm 4.4 \%$ (4).

In contrast, in the control group, the deviating rates of coefficients $(C=\mathrm{SD} /$ mean value $\times 100 \%)$ of the LDds of the neurons in the IMHV and the LPO in the NC were 25.0 and $24.6 \%, 14.5$ and $16.9 \%$ in the $\mathrm{n}-\mathrm{IC}$, and 16.3 and $17.2 \%$ in the IC, respectively. The deviation coefficients of the LDds in the memory-related nuclei were $24.8 \pm 0.3 \%$ (2) and $16.2 \pm 1.2 \%$ (4) in the $n-I C$ and IC as the control of the NC, respectively. These data showed that the deviating coefficients of the MeAstimulated chicks were significantly decreased by $34.7 \%$ $(\mathrm{t}=9.248>\mathrm{t} 0.05, \mathrm{df}=4)$. In the experimental group, the 
deviating coefficients of the LDds in the IMHV and the LPO in the NC, n-IC, and IC were 23.9 and 18.2, 24.3 and $22.2 \%$, and 28.5 and $27.8 \%$, respectively. The deviating coefficients of the LDds in the NC were $21.1 \pm$ $4.0 \%$ (2) and were $25.7 \pm 3.0 \%$ (4) in the IC. There was no significant difference between the groups. Further, compared to the control group, the deviating coefficients of the LDds in the MeA-stimulated chicks in the experimental group were increased significantly by $53.0 \%$ $(\mathrm{t}=5.510>\mathrm{t} 0.05, \mathrm{df}=6)$.

In Figure 5(2)-A and B, compared to the control group, the TLd of the neurons in the LPO in the n-IC was not significantly different, but all other TLds from each side of the IMHV in the NC, n-IC and IC and the LPO in the NC and IC were significantly decreased by $30.9 \pm 7.9 \%$ (5).

\section{DISCUSSION}

In this study, we showed that the majority of the 1-day-old chicks that were hatched from a natural geomagnetic field (control group) were able to form long-term memories in a one-trial avoidance task using MeA (Wang et al., 2003). After $12 \mathrm{~h}$ of being stimulated with MeA, both the LDds and TLd of each neuron from the two sides tested from the IMHV and the LPO in the n-IC and IC were significantly increased as compared to the NC, and the increase in both left and right sides was similar, which was consistent with previous studies ${ }^{14}$. These data indicate that by stimulating the neurons in the memoryrelated nuclei with $\mathrm{MeA}$, the dendrites and spines would undergo hyperplasia, which would allow more synaptic connections to be modulated. These changes would constitute the structural foundation of memory function in the 1-day-old chicks (Stewart and Rusakov, 1995; Barber et al., 1999). Moreover, the deviating coefficients of the LDds in the stimulated chicks were significantly decreased by $35.1 \%$ compared to the NC, and this indicates that the dendritic spines that were stimulated by MeA showed reduced "synchronous" hyperplasia. Our results are consistent with a previous study that observed the same effect in the density of dendritic spines from neurons in the memory-related nuclei isolated from 7day-old chicks subjected to auditory stimulation (Braun et al., 1999). For the few chicks that did not show long-term memory (n-IC), the LDds of the neurons from the two sides of the IMHV were significantly decreased by $10.5 \%$ compared to the IC, and the number of neurons with TLd higher than $1000 \mu \mathrm{m}$ was also decreased 1.5 fold. This indicates that although adding MeA may accelerate the rapid hyperplasia that is found in the dendrites and spines of neurons in the memory-related nuclei, the nerve network was insufficient to form long-term memory in some subjects, and these effects may be related to other changes in the synaptic structure or in the deficiency to synthesize substances that are important for creating memories (Scholey et al., 1992; Rose, 1995; Soderling and Derkach, 2000; Nguyen, 2002). Rose (1991) and Tiunova et al. (1998) have suggested that the memory flow would be L-IMHV $\rightarrow$ R-IMHV $\rightarrow$ LPO. Therefore, the long-term memory would be formed first in the IMHV and then be transferred to the LPO to be stored. Our results illustrated that the LDds and TLd of the neurons from both sides of the IMHV were increased significantly compared to the LPO, which indicates that after a $12 \mathrm{~h}$ treatment with $\mathrm{MeA}$, the long-term memory has not yet been stored in the LPO. In addition, the formation of the long-term memory that was stimulated by MeA begins by $60 \mathrm{~min}$ and can be detected as late as $24 \mathrm{~h}$ (Gao and Kuang, 1996). Further, the morphological changes in the neurons are still observed after $48 \mathrm{~h}$ (Rose and Stewart, 1999).

When we examined 1-day-old chicks that hatched from the HMFS (experimental group), we found that the chicks retained short- and medium-term memory in the one-trial avoidance task, but long-term memory was impaired (Wang et al., 2003). In the experimental group, the LDds of the neurons from both the left and right sides of the IMHV and the LPO in the NC, n-IC and IC were similar to that of the NC of the control group, whereas the NC of the control group was decreased significantly by $17.4 \%$ compared to the $\mathrm{n}-\mathrm{IC}$ and IC of the control group. With the exception of two sides in the LPO, all of the TLds (IMHV and LPO in $\mathrm{n}-\mathrm{IC}$ and IC, and LPO in NC) were decreased significantly by $30.9 \%$. Further, in the experimental group, the deviating coefficients of the LDds in the chicks stimulated with MeA did not show any significant difference compared to the NC and were significantly increased by $53.0 \%$ compared to the stimulated chicks in the control group. These results indicate that although the deprivation of geomagnetism does not effect the normal development of the dendritic spines of the neurons from the memory-related nuclei, the hyperplasia caused by MeA was inhibited, and the "synchronous" hyperplasia was lost. In addition, the development and growth of the neuronal length were significantly inhibited. Therefore, the neural network involved in long-term memory was not able to be established, and this may be the cause of the impairment to the formation of long-term memory. Additionally, the impairment may be related to other factors, such as changes in the synaptic structure or in the deficiency of molecules involved in memory (Ünal et al., 2002; Soderling and Derkach, 2000; Nguyen, 2002). It is wellknown that the NMDAR and the AMPAR in the IMHV and the LPO in 1-day-old chicks play important roles in forming and retaining new memories. The NMDAR is a voltage- and transmitting-gated diploid channel and can increase the intracellular $\mathrm{Ca}^{2+}$ level by regulating glutamate levels (Nguyen, 2002). It has been well established that $\mathrm{Ca}^{2+}$ is important in long-term potentiation (LTP) induction, and it is difficult to induce LTP in the absence of $\mathrm{Ca}^{2+}$. In contrast, abundant $\mathrm{Ca}^{2+}$ can directly induce LTP and reduce the threshold for inducing LTP, 
and LTP is the basis of learning and memory (Xu, 2000). In addition, in $\mathrm{Y}$-GABAergic synapses, moderately increasing $\mathrm{Ca}^{2+}$ leads to the growth of dendritic spines, but an excessive increase in the $\mathrm{Ca}^{2+}$ levels causes the spines to form and collapse rapidly (Segal et al., 2000). The GABA-A and GABA-B receptors have a secondary role in the induction of LTP and are important in storing memories (Weng et al., 1996). Hence, the impairment of memory formation in the 1-day-old chicks that were hatched from the HMFS may not only inhibit hyperplasia of neuronal spines following stimulation with the bitter compound, MeA, but may also cause changes in membrane structure and disrupt the normal function of channels, which would result in the inhibition of LTP. This would predict that the loss of normal geomagnetism during the development of chicken embryos may affect the normal development of membrane structure and channels of the neurons in the IMHV and the LPO. The impairment of long-term memory in 1-day-old chicks using the one-trial avoidance task may be recovered. In Drosophila that were housed continuously in the HMFS, the ability to learn and remember was seriously impaired, but by removing the flies from the HMFS and housing them in conditions of natural geomagnetism for six generations, the ability to form long-term memories was restored (Zhang et al., 2004). In addition, the memory of chicks that hatched from the HMFS did not show any deficiency in long-term memory after being bred in normal space.

Some of the chicks that were hatched from the HMFS displayed long-term memory by exhibiting the avoidance reaction, but the density of dendritic spines in the neurons from the memory-related nuclei was similar to the chicks not showing an avoidance reaction after being stimulated with MeA. In addition, the TLd in the IMHV was increased. The lack of difference between the 2 groups may be explained by the length of the observation time because previous studies have shown that the number of dendritic spines was decreased gradually from the original peak after being trained (Stewart and Rusakov, 1995). In rats, the density of spines increased at $3 \mathrm{~h}$ after being trained, reached the maximum density at $6 \mathrm{~h}$, and then decreased to basal levels (Ünal et al., 2002). Therefore, it is important to measure the density of dendritic spines at different time points after training. In contrast, long-term memory may not be dependent on the changes in the synaptic structure, and it is possible that some other neural-chemistry processes, such as the synthesis of $\alpha$-tubulin and glycoprotein, compensated for the loss of function so that the long-term memory could be formed.

In rats, the LDd of pyramid cells in the cortex was 0.60 $\pm 0.21 \mu \mathrm{m}$ (in 42 dendrite segments, $50 \mu \mathrm{m}$ per segment), and the deviation coefficients were $35.0 \%$ (Peter and Kaiserman-Abramof, 1970). In the control group of another study that examined 7-day-old chicks, the LDds of large neurons from the dorsocaudal neostriatum were
$0.47,0.86$ and 0.97 to $1.00 \mu \mathrm{m}$, and the deviation coefficients were $21.3,14.0$ and 10.2 to $13.0 \%(n=5)$. In the experimental group that was imprinted by stimulating with sound, the LDds were $0.19,0.54$ and 0.69 to 0.76 $\mu \mathrm{m}$, and the deviation coefficients were 21.5, 13.0 and 2.7 to $5.8 \%$ (Braun et al., 1999). From these data, we can conclude that our results for the LDds are reliable. By depriving chicks of the geomagnetic field space that is normally used by all animals throughout evolution, the magnetism balance in their bodies may be eliminated, thereby affecting the motion and function of electrical particles. Previous data had indicated that the manual weak magnetic field could influence the conformation, structure and holistic behavior of the central nervous system ( $\mathrm{Li}, 1994)$. A prolonged exposure to the HMFS could not only inhibit the growth and development of many different types of plants (Dubrov, 1978), arouse gene mutation related to the albinism of hairs and significantly reduce the content of some neurotransmitters (Li et al., 1999, 2001), but could also lead to a significant alteration in the formation of chromosomes in human lymphocytes and fibroblasts (Belyaev et al., 1997). In this vein, deprivation of geomagnetism during the development of chicken embryos could negatively affect brain function. Therefore, our study may not only offer some evidence for the effect of geomagnetic deprivation on the activity of humans in outer space (ether biology) but may also suggest a possible relationship between geomagnetism and the evolution of species.

\section{ACKNOWLEDGEMENTS}

The authors would like to send appreciation to Prof. Tao Song in Institute of Electrical Engineering, Chinese Academy of Sciences for guidance and help. This work was authenticated by the National Natural Science Foundation of China (Grant No. 30400090).

\section{REFERENCES}

Barber TA, Howorth PD, Klunk AM, Cho CC (1999). Lesions of the intermediate medial hyperstriatum ventral impair sickness conditioned learning in day-old chicks. Neurobiol. Learn. Mem. 72:128-141.

Belyaev IY, Alipov YD, Harms-Ringdahl M (1997). Effect of zero magnetic field on the conformation of chromatin in human cells. Biochim. Biophys. Acta 1336:465-473.

Braun K, Bock J, Metzger M, Jiang S, Schnabel R (1999). The

dorsocaudal neostriatum of the domestic chick: a structure serving higher associative functions. Behav. Brain Res. 98:211-218.

Dubrov AP (1978). The Geomagnetic Field and Life, Geomagnetobiology. Plenum Press, New York. pp. 92-112.

Gao Y, Kuang PZ (1996). The research progress on the forming mechanism of memory in some different learning models. Psychol. Trends 4:7-10 (in Chinese).

Gibbs ME, Ng KT (1976). Memory formation: A new three-phase model. Nerurosci. Lett. 2:165-169.

Jiang J, Jin H, Lin Y, Chen H, Yang X, Zeng X, Zhou X (1998). 
The effect of magnetic field-free space on the acoustic behavior of budgerigars (Melopsittacus undulafus). Acta Seismol. Sin. 11:487493.

Jiang J, Wang X, Xu M (2003). An experimental system for research the biological effects of hypomagnetic field space. Acta Biophys. Sin. 19:218-221 (in Chinese).

Li GD (1994). The progress in biomagnetics in foreign. J. Chinese Med. 6:50 (in Chinese).

Li J, Wu Q, Wang Q, Jiang J, Jin $H$, Lin $Y$ (2001). Effect of magnetic free field space (MFFS) on GABA, glycine and taurine of cortex, cerebellum and Basilar Nucleus in hamster. Prog. Biochem. Biophys. (in Chinese) 28:358-361.

Li JF, Wu QJ, Wang Q, et al (1999). Magnetic field-free space and the albinism of hamster. Chinese Sci. Abstracts (in Chinese) 5:781-783.

Lovely RH, Creim JH, Miller DL (1992). Exposure to magnetic fields during spatial learning increases error rates in rats: early studies, The First World Congress for Electricity and Magnetism in Biology and Medicine, Abstract Book, Florida, Lake Buena Vista, Buena Vista Palace. p. 23.

Nguyen PV (2002). Protein synthesis during LTP: linking synaptic activity to translation. Trends Neurosci. 25:180.

Peters A, Kaiserman-Abramof IR (1970). The sall pyramidal neuron of the rat cerebral cortex. The perikaryon, dendrites and spines. Am. J. Anat. 127:321-355.

Rose SPR (1991). How chicks make memories: The cellular cascade from $c$-fos to dendritic remodeling. Trends Neurosci., 14: 390-396.

Rose SPR, Stewart MG (1999). Cellular correlates of stages of memory formation in the chick following passive avoidance training. Behav. Brain Res. 98:237-243.

Rose SPR (1995). Cell-adhesion molecules, glucocorticoids and longterm memory formation. Trends Neurosci. 18:502-506.

Rusakov DA, Stewart MG (1995). Quantification of dendritic spine populations using image analysis and a tilting disector. J. Neurosci. Methods 60:11-21.

Rusakov DA, Stewart MG, Davies HA, Harrison E (1993). Spatial rearrangement of the vesicle apparatus in forebrain synapses of chicks 30 min after passive avoidance training. Neurosci. Lett.154: 13-16.

Scholey AB, Bullock S, Rose SPR (1992). Passive avoidance learning in the young chick results in time- and locus-specific elevations of alpha-tubulin immunoreactivity. Neurochem. Int. 21:343-350.

Scholey AB, Rose SPR, Zamani R, Bock E, Schachner M (1993). A role for the neural cell adhesion molecule in a late, consolidating phase of glycoprotein synthesis six hours following passive avoidance training of the young chick. Neuroscience 55:499-509.
Segal M, Korkotian E, Murphy DD (2000). Dendritic spine formation and pruning: common cellular mechanisms. Trends Neurosci. 23:53-57.

Soderling TR, Derkach VA (2000). Postsynaptic protein phosphorylation and LTP. Trends Neurosci. 23:75-80.

Sojka M, Davies HA, Rusakov DA, Stewart MG (1995). 3-Dimensional morphometry of intact dendtritic spines observed in thick sections using an electron microscope. J. Neurosci. Methods 62:73-82.

Squire LR, Knowlton B, Musen G (1993). The structure and organization of memory. Ann. Rev. Psychol. 44:453-495.

Stewart MG, Lowdnes M, Hunter A, Doubell T (1992). Memory storage in chicks involves an increase in dendritic spine number and synaptic density. Brain Dysfunct. 5:50-64.

Stewart MG, Rusakov DA (1995). Morphylogical changes associated with stages of memory formation in the chick following passive avoidance training. Behav. Brain Res. 12:21-28.

Tiunova AA, Anokhin KV, Rose SPR (1998). Two critical periods of protein and glycoprotein synthesis in memory consolidation for visual categorization learning in chicks. Learn Mem. 4:401-410.

Ünal B, Bradley PM, Sahin B, Canan S, Aslan H, Kaplan S (2002). Estimation of numerical density and mean synaptic height in chick hippocampus 24 and 48 hours after passive avoidance training. Dev. Brain Res. 136:135-144.

Wang XB, Xu ML, Li B, Li DF, Jiang JC (2003). Long-term memory was impaired in one-trial passive avoidance task of day-old chicks hatching from hypomagnetic field space. Chinese Sci. Bull. 48:24542457.

Weng XC, Kuang PZ, Chen SS (1996). Y-GABA energic system and memory: the research progress in behavior and pharmacology. Psychol. Trends (in Chinese) 4:19-22.

Xu K (2000). Essential of neurobiology, Science Publishing Company, Beijing, (in Chinese).pp. 312-329 Youngren OM, Phillips RE (1978). A stereotaxic atlas of the brain of the three-day-old domestic chick. J. Comp. Neurol. 181:567-599.

Zhang B, Lu HM, Xi W, Zhou XJ, Xu SY, Zhang K, Jiang JC, Li Y, Guo AK (2004). Exposure to hypomagnetic field space for multiple generations causes amnesia in Drosophila melanogaster. Neurosci. Lett. 371:190-195. 